\title{
Structural Elucidation of Dendritic Host-Guest Complexes by X-ray Crystallography and Molecular Dynamics Simulations
}

\author{
Theresa Chang, ${ }^{[a]}$ Koen Pieterse,${ }^{[b]}$ Maarten A. C. Broeren ${ }^{[a]}$ Huub Kooijman, ${ }^{[c]}$ \\ Anthony L. Spek, ${ }^{[\mathrm{cl}]}$ Peter A. J. Hilbers, ${ }^{[\mathrm{b}]}$ and E. W. Meijer ${ }^{*[a]}$
}

\begin{abstract}
The multiple monovalent binding of adamantyl-urea poly(propyleneimine) dendrimers with carboxylic acid-urea guests was investigated using molecular dynamics simulations and $\mathrm{X}$ ray crystallography to better understand the structure and behavior of the dynamic multivalent complex in solution. The results from the two methods are consistent and suggest a preferred molecular picture of this complicated
\end{abstract}

aggregate of multiple components. The guest molecules can bind to the dendrimer in a variety of ways although most involve hydrogen-bonding interactions between urea groups of the dendrimer

Keywords: computer chemistry dendrimers - host-guest systems • molecular dynamics structure elucidation with urea and/or carboxylic acid groups of the guest. In addition, acid-base interactions between the carboxylic acid of the guest and the tertiary amine in the interior of the dendritic host are present. Our proposed structure gives important information about the predominant dynamic interactions between the host and guest and illustrates how they fit together and interact with each other.

\section{Introduction}

Multiple monovalent interactions are the simultaneous association of multiple ligands of one molecule or complex to multiple binding sites on a given receptor and are important in numerous biological processes such as cell recognition events. Multiple interactions often have enhanced properties that are significantly different from the properties of their constituent monovalent ligands. An effective way of achieving a multivalent display of ligands is by attaching the ligands of interest to the periphery of dendrimers. ${ }^{[1-4]}$ We have previously reported the functionalization of adaman-

[a] Dr. T. Chang, Dr. M. A. C. Broeren, Prof. E. W. Meijer

Laboratory of Macromolecular and Organic Chemistry

Eindhoven University of Technology

PO Box 513, 5600 MB Eindhoven (The Netherlands)

Fax: $(+31) 40-245-1036$

E-mail: e.w.meijer@tue.nl

[b] Dr. K. Pieterse, Prof. P. A. J. Hilbers

Biomodeling and Bioinformatics

Eindhoven University of Technology

PO Box 513, 5600 MB Eindhoven (The Netherlands)

[c] Dr. H. Kooijman, Prof. A. L. Spek

Bijvoet Center for Biomolecular Research Crystal Structural Chemistry, Utrecht University Padualaan 8, $3584 \mathrm{CH}$ Utrecht (The Netherlands)

Supporting information for this article is available on the WWW under http://www.chemeurj.org/ or from the author. tyl-urea dendrimers using noncovalent interactions resulting in a statistical, yet adaptable, distribution of ligands at the periphery of the dendrimer scaffold. ${ }^{[5]}$

The adamantyl-urea dendrimers, made by reacting adamantyl isocyanate with poly(propyleneimine) dendrimers, were designed to bind to acid-urea guests via a combination of electrostatic and hydrogen-bonding interactions (Scheme 1). Upon mixing, proton transfer would occur from the carboxylic acid of the guest to the tertiary amine of the pincer and the two would be held together by electrostatic (ion pairing) interactions. The complex would be further stabilized by urea-urea hydrogen bonds with the guest sandwiched between the urea groups of the pincer-like moiety. The urea moieties of the dendrimer were appended with bulky adamantyl groups to reduce intramolecular hydrogen bonding of urea groups within pincers, but they should bind efficiently to guest molecules with less bulky side chains.

Many experiments showed spectroscopic evidence for the depicted design, although a static picture can never visualize in a reasonable way a highly dynamic multicomponent system. Like the components of a cell or the arrangement of surfactants in micelles and vesicles, the dendrimer-guest complex is neither rigid nor static and the noncovalent interactions holding the components together are constantly broken and reformed. The spherical and dynamic nature of dendrimers makes single crystal studies limited to the lower generations. $^{[6]}$ Molecular dynamics simulations of dendrim- 


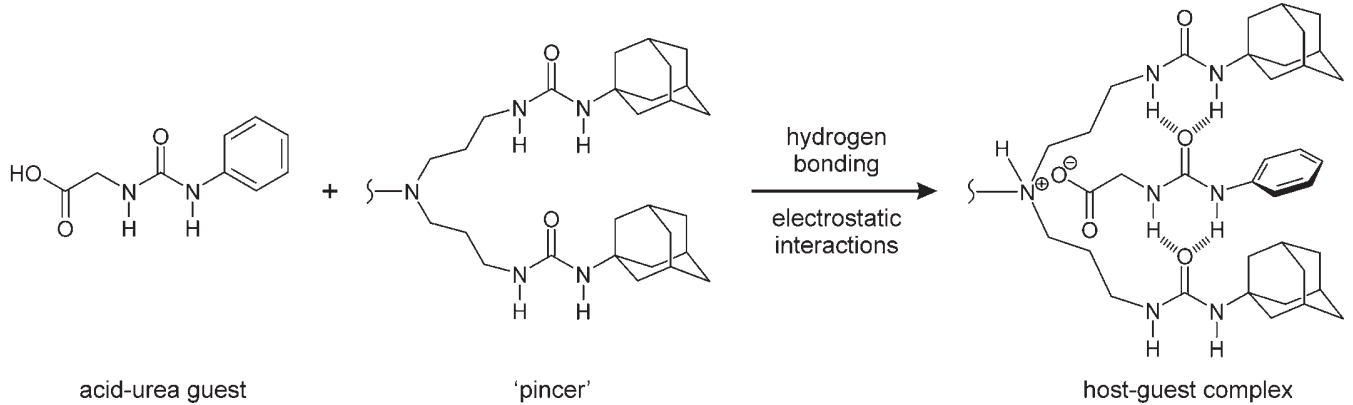

Scheme 1. Binding of an acetic acid-urea guest to a dendrimer.

ers are often focused on the overall shape, density profile and positions of the end groups. ${ }^{[7]}$ Specific supramolecular attention in the simulation of dendrimers has been limited to encapsulated host-guest systems. ${ }^{[8]}$ In this paper, we wish to present an accurate representation of this dynamic hostguest complex, based on analyses of the crystal packing structures of model compounds and atomistic molecular dynamics simulations. Our proposed structure will give important information about the predominant dynamic interactions between the host and guest and illustrate how they fit together and interact with each other.

\section{Results}

X-ray crystallography: ${ }^{[9]}$ Since crystals of dendritic structures were difficult to come by, we have obtained X-ray quality single crystals of what we thought are the relevant binding sites of the dendrimer, the pincer-like structures ${ }^{[10]}$ with adamantyl and phenyl groups ( $\mathbf{1}$ and $\mathbf{2}$, respectively; see Supporting Information for preparation of $\mathbf{1}$ ), as well as guest molecules $^{[11]}$ with the same end groups $(\mathbf{3}, 3$.TEA, and 4) shown in Figure 1. Co-crystals of pincer-guest complexes were also attempted ${ }^{[12]}$ but without success, suggesting that a guest sandwiched between the arms of a pincer is not necessarily a favored (stable) structure in a crystalline lattice.

The packing structures of the adamantyl pincer show that the pincer is quite flexible (Figure $2 a$ and $b$ ). Two polymorphs were found in crystals grown from the same solvent system. These packing structures show that the adamantyl group is bulky enough to prevent intra-pincer hydrogen bonding between urea groups. Hydrogen bonding is ob- served between pincers, forming infinite hydrogen-bond stacks in both polymorphs with urea groups rotated by $90^{\circ}$ relative to each other. A pincer without bulky groups shows efficient intra-pincer hydrogen bonding as illustrated in Figure $2 \mathrm{c}$. The phenyl pincers are also more difficult to dissolve in organic solvents, exemplifying the difficulty in breaking these intra-pincer hydrogen bonds to insert a guest.

A phenyl-terminated guest molecule 3 results in a columnar packing structure (Figure 3a) that is reminiscent of that of the phenyl pincer 2. An infinite hydrogen-bonding stack is formed with guest molecules rotated $180^{\circ}$ relative to the ones above and below. Acid-urea [O- $\mathrm{H} \cdots \mathrm{O}]$ hydrogen bonds link these columns together (Figure $3 \mathrm{~b}$ ), however, no $\pi-\pi$ stacking interactions were observed with either pincer 2 or guest 3. The crystal structure of the adamantyl guest (Figure $3 \mathrm{c}$ and $\mathrm{d}$ ) is in agreement with what was observed previously. The bulky adamantyl groups prevented urea-urea bonds so that hydrogen bonds are formed between the urea moieties with carboxylic acids of adjacent guest molecules instead.

The triethylammonium salt of the guest $\mathbf{3}$ was recrystallized to probe acid-base interactions present in the dendrimer-guest complex. In this crystal structure (Figure 4) we observed that only half of the carboxylic acids are deprotonated, a common occurrence for weak acids in the solid state ${ }^{[13]}$ as a strong $\left[\mathrm{O}-\mathrm{H} \cdots \mathrm{O}^{-}\right]$hydrogen bond is formed. Urea-urea hydrogen bonds are not present in this crystal structure because of the possibility of ion-dipole interactions, which are usually stronger ${ }^{[14]}$ than dipole-dipole (hydrogen bonding) interactions. We can conclude from the crystal structures shown above that there are many more types of interactions available to join dendrimer and acid-
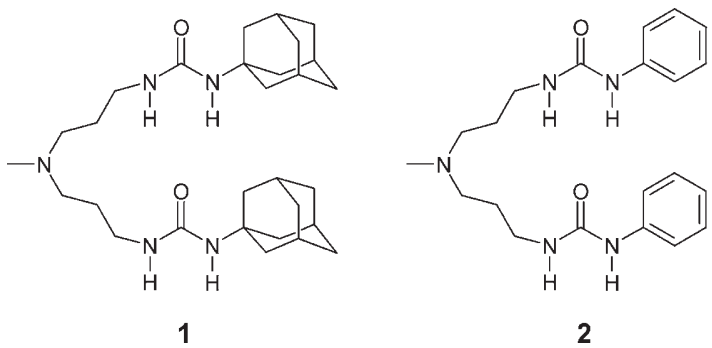<smiles>O=C(O)CNC(=O)Nc1ccccc1</smiles>

3<smiles>O=C(O)CNC(=O)NC12CC3CC(CC(C3)C1)C2</smiles>

4

Figure 1. Structures investigated using X-ray crystallography. 
a)

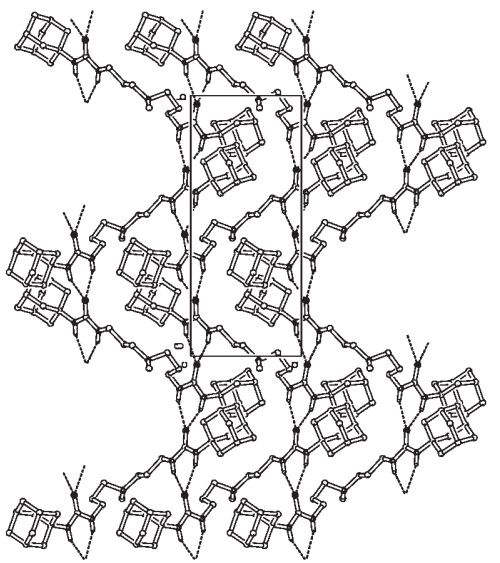

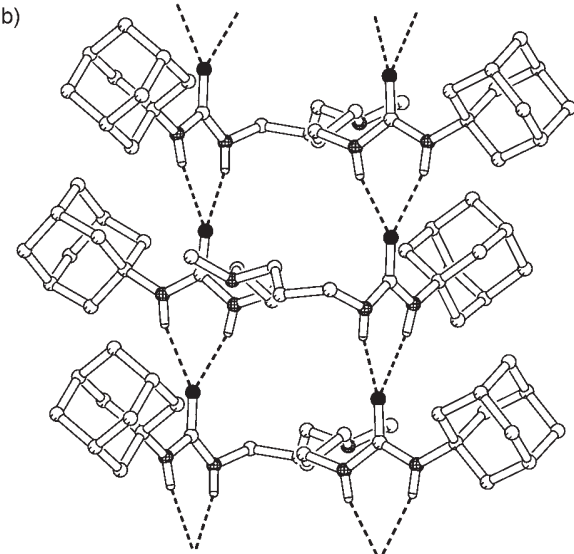

c)

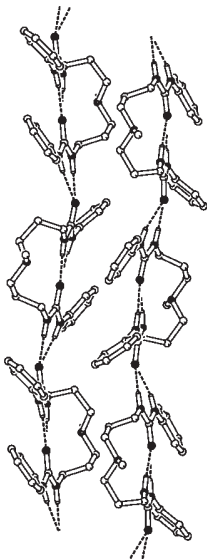

Figure 2. Solid-state superstructure of a,b) two polymorphs of the adamantyl pincer and c) the phenyl pincer.

a)

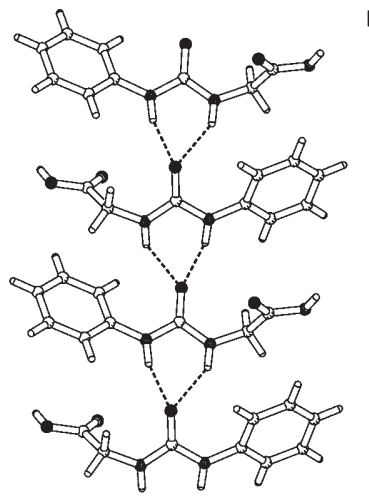

b)

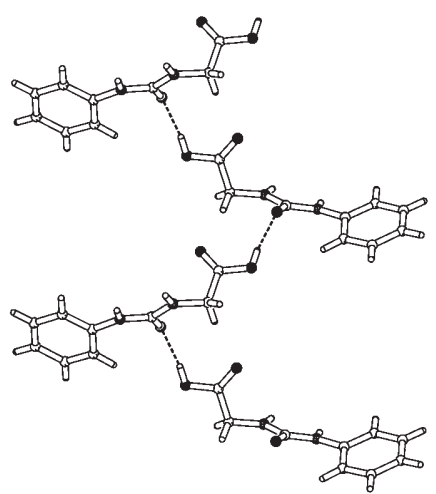

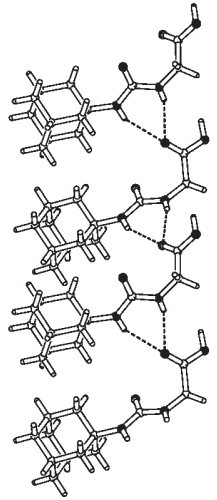

d)

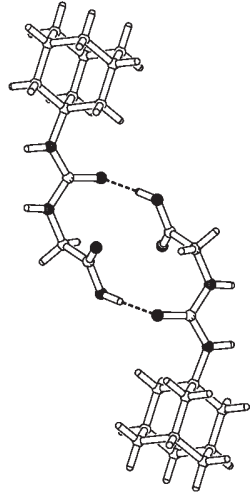

Figure 3. a) Phenyl guests form infinite stacks via urea-urea hydrogen bonding and b) the stacks are linked to other stacks via acid-urea bonds. Adamantyl-urea guests form columns via c) [ $\mathrm{N}-\mathrm{H} \cdots \mathrm{O}]$ urea acid hydrogen bonding within the column and d) $[\mathrm{O}-\mathrm{H} \cdots \mathrm{O}]$ acid-urea hydrogen bonding between stacks to the carboxylic acid groups.

a)

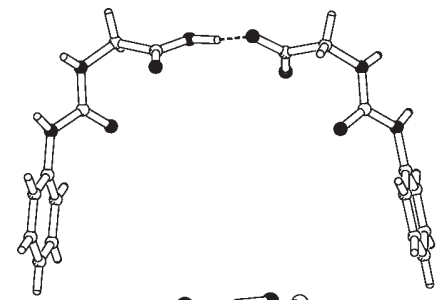

b)

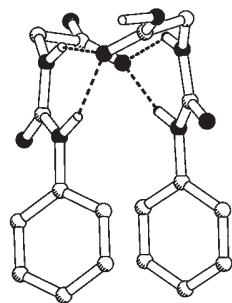

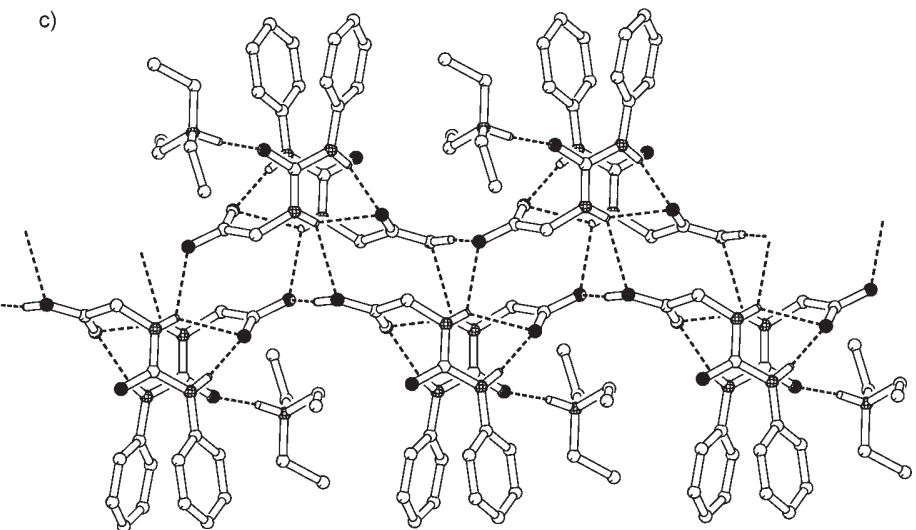

Figure 4. X-ray crystal structure of 3.TEA shows the formation of a) a protonated-unprotonated acid pair linked together with an ion-dipole $\left[\mathrm{O}-\mathrm{H}^{-\cdots} \mathrm{O}^{-}\right]$ interaction as well as b) $\left[\mathrm{N}-\mathrm{H}^{\cdots} \mathrm{O}^{-}\right]$hydrogen bond. The overall packing structure is composed of the aforementioned interactions as well as ammonium ion-urea interactions (c).

urea guests in addition to the carboxylate/ammonium and urea-urea hydrogen-bonding interactions illustrated in Scheme 1.
Molecular dynamics simulations: Using NAMD $2.5,^{[15]}$ molecular dynamics (MD) simulations were performed on the 3rd- and 5th-generation dendrimers shown in Figure 5 (8 

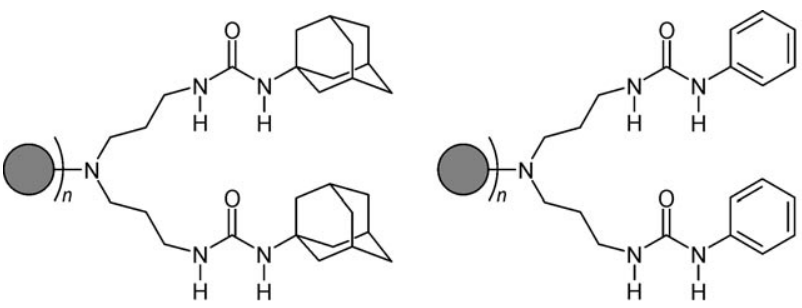

D1a: $n=8$

D1b: $n=32$
D2a: $n=8$

D2b: $n=32$
Figure 5. Structures investigated using MD simulations.

and 32 pincer-like moieties, respectively) having adamantyl (D1a, D1b) or phenyl groups (D2a, D2b) at the periphery. The dendrimers were analyzed both with and without acidurea guests $\mathbf{5}$. The dendrimers were simulated for 2 ns using $1 \mathrm{fs}$ time steps, and atom coordinates and velocities were sampled every picosecond. In simulations that included acid-urea guests ( 8 and 32 guests for 3rd- and 5th-generation dendrimers, respectively) initial structures were generated in $1 \mathrm{~ns}$ pre-simulations from extended dendrimer structures (for details see Supporting Information). Guest molecules were uniformly distributed on the surface of a sphere, tightly enclosing these dendrimers. For simulations that only involved the dendrimer, we used just the extended dendrimer structures as a starting point. These extended structures quickly collapse to form a more compact structure as favorable van der Waals interactions and hydrogen bonds (by means of electrostatic interactions) are formed between different sections of the macromolecule/complex. In each simulation, a starting temperature of $500 \mathrm{~K}$ was used and at this temperature noncovalent bonds could still be broken and reformed. The temperature was gradually decreased to $300 \mathrm{~K}$ in the first nanosecond and remained at $300 \mathrm{~K}$ during the last nanosecond of the simulations. As the temperature decreases, the conformations of the dendrimers are locked, as the system no longer has enough energy to break hydrogen bonding and/or acid-base interactions. The acid-base reactions of these guests with the basic dendrimers were simulated as acid-base interactions (i.e., electrostatic interactions) consistent with the gas phase. ${ }^{[16]}$

As shown in Figure 6, 3rd-generation adamantyl- and phenyl-urea dendrimers, D1a and D2a, do not exhibit a globular structure. The groups at the periphery (adamantyl or phenyl) tend to cluster on one side of the molecule to maximize the number of urea-urea hydrogen-bonding interactions, leaving the interior of the dendrimer as well as the diaminobutane core exposed. Addition of guests improves the surface coverage, resulting in a more spheroid shape, however, still having an accessible dendrimer interior. Fifth generation dendrimers (D1b, D2b) are more globular in shape although patches of dendrimer interior are still exposed. In some cases, even the diaminobutane core is still exposed on these dendrimers. The addition of guests 5 to 5 th-generation dendrimers gave structures with only small portions of the dendrimer interior appearing at the surface.<smiles>O=C(O)CNC(=O)NCc1ccccc1</smiles>
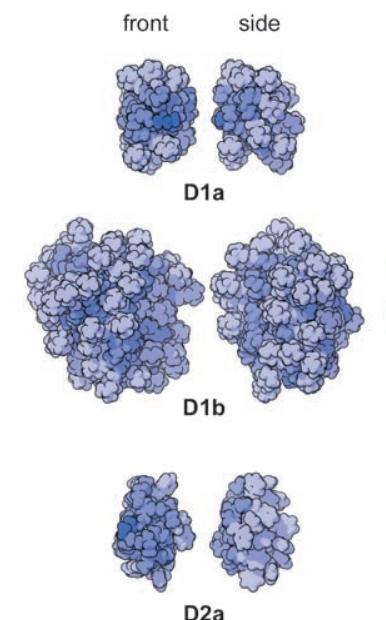

D2a

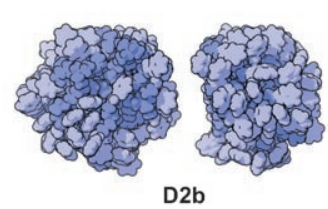

D2b
The diaminobutane core of the dendrimers is situated in the center of the aggregates. Overall, the bulky end groups of the adamantyl-terminated dendrimers result in more crowding at the periphery compared with the phenyl-terminated dendrimers.

The driving force for the formation of each of the structures

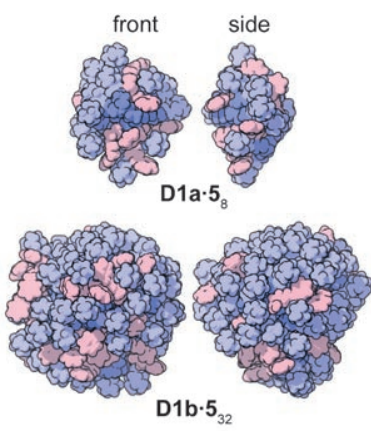

Figure 6. Structure of the dendrimers and dendrimer complexes after a 2 ns simulation. The dendrimers are depicted in blue (darker blue for the interior), while the guests are colored pink. For each structure a front and side view is shown.

mentioned above is the stabilizing electrostatic interactions arising for the most part from hydrogen-bonding and acidbase interactions. Hydrogen bonds between urea groups make up a majority of these stabilizing forces because of both i) the large number of urea groups present in either the dendrimer or dendrimer-guest complex and ii) the strength of these bifurcated hydrogen bonds. The hydrogen-bonding interactions between the urea groups of the dendrimer and guest molecules were analyzed using the atom trajectories obtained from the molecular dynamics simulations outlined in Table 1. The urea-urea hydrogen-bonding interactions analyzed are defined as consisting of two hydrogen bonds where the distances between the hydrogen atoms of the donor and the oxygen atom of the acceptor are $2.5 \AA$ or less. Hence, the total number of hydrogen-bonding interactions possible is the same as the number of urea groups. Even though nearly all the urea groups participate in hydrogen-bonding interactions, only about $45 \%$ of these are bifur- 
Table 1. Urea-urea hydrogen-bonding distributions in dendrimer/dendrimer complexes.

\begin{tabular}{llccc}
\hline $\begin{array}{l}\text { Dendrimer } \\
\text { structure }\end{array}$ & $\begin{array}{l}\text { Urea } \\
\text { linkages [\%] }{ }^{[\mathrm{a}]}\end{array}$ & \multicolumn{3}{c}{ Urea linkages present } \\
& & $\begin{array}{c}\text { within } \\
\text { dendrimer [\%] }\end{array}$ & $\begin{array}{c}\text { between } \\
\text { dendrimer } \\
\text { and guests [\%] }\end{array}$ & $\begin{array}{c}\text { between } \\
\text { guests [\%] }\end{array}$ \\
\hline D1a & 43 & 100 & - & - \\
D1 a·5 & 45 & 48 & 48 & 4 \\
D1b & 48 & 100 & - & - \\
D1b·5 & 47 & 45 & 46 & 9 \\
D2a & 42 & 100 & - & - \\
D2a.5 & 49 & 52 & 45 & 3 \\
D2b & 46 & 100 & - & - \\
D2b·5 & 43 & 49 & 42 & 9 \\
\hline
\end{tabular}

[a] Given as the percentage of possible urea linkages. [b] Subdivision of the percentage of urea linkages per linkage type.

cated out of all possible linkages in all dendrimers with or without acid-urea guests. The remainder participate either in longer distance hydrogen-bonding interactions with urea or carboxylate groups or interact only with one hydrogen bond.

The presence or absence of guest molecules does not change the percentage of urea-urea hydrogen-bonding interactions in the macromolecule/complex. As a result of steric issues arising from bulky adamantyl groups, urea-urea bonds found in simulations regarding adamantyl-urea dendrimers (with or without guests) exhibit torsion angles of around $90^{\circ}$ (perpendicular orientation) as was found in all crystal structures containing adamantyl urea groups. In contrast, there was no preferential urea-urea torsion angle found in simulations pertaining to phenyl dendrimers.

In dendrimer host-guest complexes, about $45 \%$ of all the urea-urea bonds formed are between dendrimer and guests, signifying the full integration of guest molecules into these dendrimers. Approximately $50 \%$ of urea linkages are formed between urea groups within dendrimers, presumably because they constitute $67 \%$ of urea groups in the complex. Hydrogen-bonding interactions of this type between guest molecules are small in most cases (3-9\%), with a tendency to be larger in case of the fifth-generation dendrimers.

Acid-base interactions between the tertiary amine groups of the dendrimer and the carboxylic acids of the guests are also important for the complexation event and are the second stabilizing interaction holding the guest and dendrimer together. In the gas phase, proton transfer is only likely between strong acids and strong bases and, hence, in these dendrimer-guest complexes acid-base interactions should exist as hydrogen bonds between two polarized groups rather than an ion pair. Since tertiary amines are present in all layers of the dendrimer (even at the core), it is conceivable that guest molecules can bind to amines in the interior of the dendrimer as well as with those near the periphery. However, acid-urea guests are mainly found near the periphery of the dendrimer (Table 2). The guests interact with amines at the outer layers of the dendrimer because there are more tertiary amines available and to maximize the
Table 2. Number and location of acid-base interactions in dendrimerguest complexes.

\begin{tabular}{lclllll}
\hline $\begin{array}{l}\text { Dendrimer struc- } \\
\text { ture }\end{array}$ & $\begin{array}{c}\text { Acid-base } \\
\text { interactions } \\
{[\%]^{[\mathrm{a}]}}\end{array}$ & \multicolumn{5}{c}{$\begin{array}{c}\text { Tertiary amines involved } \\
\text { in acid-base interactions }\end{array}$} \\
& & $\alpha$ & $\beta$ & $\gamma$ & $\delta$ & $\varepsilon$ \\
& {$[\%]$} & {$[\%]$} & {$[\%]$} & {$[\%]$} & {$[\%]$} \\
\hline D1 a.5 & 42 & 20 & 20 & 60 & - & - \\
D1b·5 & 36 & 6 & 3 & 12 & 22 & 57 \\
D2 a.5 & 22 & 0 & 37 & 63 & - & - \\
D2 b.5 & 38 & 0 & 3 & 8 & 16 & 74
\end{tabular}

[a] Given as the percentage of possible acid-base interactions. [b] Subdivision of the percentage of acid-base interactions per location of the involved tertiary amines, where $\alpha-\varepsilon$ represent each successive dendrimer shell ( $\alpha$ being the dendrimer core).

number of other stabilizing interactions (including ureaurea hydrogen bonds) in the complex.

The urea-urea hydrogen bond and acid-base interactions mentioned in the pincer model only accounts for two out of the eight possible combinations of donor/guest interactions. In a dendrimer-guest complex, there are two types of hydrogen bond donors, urea $\mathrm{N}-\mathrm{H}$ and acid $\mathrm{O}-\mathrm{H}$ and they can form hydrogen bonds with all four acceptors, urea $\mathrm{C}=\mathrm{O}$, acid $\mathrm{C}=\mathrm{O}$, acid $\mathrm{O}-\mathrm{H}$ as well as the tertiary amines of the dendrimer. Hydrogen-bonding interactions with the acid $\mathrm{O}_{-}$ $\mathrm{H}$ as acceptor are negligible for the most part. However, all of the $\mathrm{C}=\mathrm{O}$ groups of carboxylic acid groups of guest molecules are involved in hydrogen-bonding interactions, the majority of them $(\approx 80 \%)$ with urea $\mathrm{N}-\mathrm{H}$ groups and the rest with acid $\mathrm{O}-\mathrm{H}$ groups of another guest. In addition, bifurcated urea-urea hydrogen bonds only account for $\approx 80 \%$ of all urea-urea hydrogen bonds in the dendrimer-guest complexes.

It is noteworthy that the behavior of the 5th-generation phenyl dendrimer complex is, in most respects, very similar to that of the analogous adamantyl dendrimer complex. The main difference between the two structures is the urea-urea hydrogen bond torsion angles. The adamantyl dendrimers and complexes exhibit strong preference for orthogonal bifurcated hydrogen bonds while the torsion angles of the phenyl dendrimers and complexes appear to be randomly distributed. The latter seems contradictory to the preferential co-planar or perpendicular orientation of urea-urea bonds, ${ }^{[17]}$ but is the direct result of modeling hydrogen bonds through electrostatic interactions (which lack directionality). Hence, the perpendicular orientation of the ureaurea linkages in adamantyl-functionalized dendrimers can solely be attributed to steric repulsions.

\section{Discussion}

Both the crystal structures and the molecular dynamics simulations clearly show that binding of the acid-urea guests to the dendrimer via the mechanism proposed in Scheme 1 is unlikely. The chaotic nature of the host-guest complex, however, makes it difficult to visualize what exactly is happening 
in the dendrimer. There are many feasible ways a guest molecule can bind to the dendrimer, often via multiple interactions to different parts of the dendrimer, and sometimes even to other guests. In fact, all the secondary interactions found in the crystal structures are also present in the MD simulations and conceivably contribute to the overall stability as well as complexity of the system. The examples shown in Figure 7 exemplify the typical interactions found in a dendrimer-guest complex. guest molecules bound to the dendritic host. For the complex, the urea groups will provide most of the stabilizing interactions from participating in urea-urea $[\mathrm{N}-\mathrm{H} \cdots \mathrm{O}]$, acidurea $[\mathrm{O}-\mathrm{H} \cdots \mathrm{O}]$ and urea-acid $[\mathrm{N}-\mathrm{H} \cdots \mathrm{O}]$ hydrogen bonds. The adamantyl substituted urea groups will give bifurcated urea-urea hydrogen bonds with the urea groups aligned orthogonal to each other, whereas a less bulky group will lead to a more random conformation. When the dendrimer or guest molecules contain phenyl groups, they might participate in $\pi-\pi$ stacking interactions. Proton transfer might occur between the acid groups of the guests and the tertiary amines of the dendrimer, depending on the solvent's ability to stabilize the newly formed ion pair.

Although it cannot do justice to the three dimensional, intricate and dynamic nature of the dendrimer-guest complexes, Figure 8 summarizes the most important interaction types found in a dendrimer-guest complex in a two dimensional
Figure $7 \mathrm{a}$ shows that guest molecules sometimes do bind to the pincer-like moiety in the dendrimer as designed, sandwiched between two arms of a pincer, although not exactly in the way shown in Scheme 1. In this example, a guest molecule forms urea-urea hydrogen bonds with one arm of a pincer, while the carbonyl group of the carboxylic acid moiety binds to the urea group of the other arm. It also binds, via an acid-base interaction, to a tertiary amine of a neighboring branch.

Guest molecules can interact favorably with other guest(s) in addition to its links with the dendrimer. In Figure $7 \mathrm{~b}$, the central guest molecule has urea-urea hydrogen bonds with two other guests. Furthermore, its carboxylic acid group has two additional interactions: an acid-base interaction with a tertiary amine and a hydrogen bond with the acidic proton of the guest below.

Often, a hydrogen-bond stack found in many of the crystal structures composed of urea moieties can be found in the dendrimer-guest complex as shown in Figure 7c. Here we see a somewhat curved urea stack, composed of alternating guests and pincer arms, each time the molecule rotates relative to the urea stack as a result of either steric reasons or reorientation of the molecule to optimize interactions with another part of the dendrimer.

Considering the results found in the crystal structures and MD simulations, what will the dendrimer-guest complexes look like in solution? Of course, the structure of the complex will be dependent on the polarity of the solvent, but will be less condensed than the crystal or simulated structures as a result of competitive van der Waals and electrostatic interactions with the solvent molecules. While obviously, the association constant will determine the number of

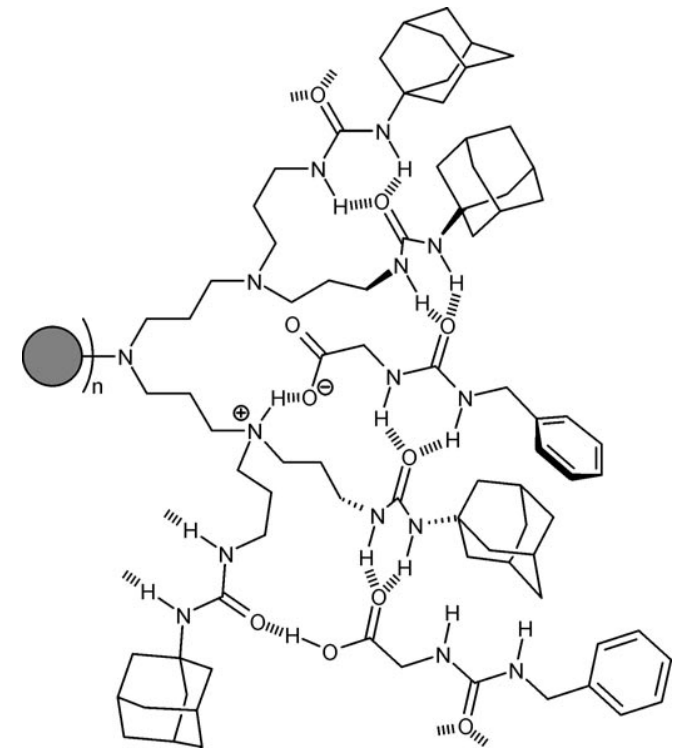

Figure 8. Proposed dendrimer complex structure in solution, showing the predominant interaction types between dendrimer and guest.

representation. Moreover, the structure presented here, based on X-ray of models and simulations, is in full agreement with earlier spectroscopic and mass spectrometry data as well as light scattering.

\section{Conclusion}

The dendrimer-guest systems studied here are dynamic and these host-guest aggregates cannot be easily represented 
with a two- or even three-dimensional static picture. We have used crystal structures of model compounds and molecular dynamics simulations to try to understand how guests occupy the "binding sites" in adamantyl-urea dendrimers. We have found that there is not one clear way that guest molecules bind to the dendrimer although they do share some common characteristics and usually form multiple interactions with different parts of the dendrimer. We have proposed a preferred structure that is in agreement with all reported spectroscopic data. Since, dendrimers are gaining more and more interest as carriers for (bioactive) guests, the presented studies is of relevance for all dendritic guest-host systems and other multiple monovalent interacting systems so often observed in Nature and nanotechnological applications.

\section{Acknowledgements}

This work was supported in part by the Council for the Chemical Sciences of the Netherlands Organization for Scientific Research (CW-NWO) and in part by the EC Sixth Framework Programme (as part of the STREP Biomach, contract \#505 487-1).

[1] For some reviews on supramolecular chemistry of dendrimers see: a) D. K. Smith, A. R. Hirst, C. S. Love, J. G. Hardy, S. V. Brignell, B. Huang, Prog. Polym. Sci. 2005, 30, 220-293; b) P. J. Gittins, L. J. Twyman, Supramol. Chem. 2003, 15, 5-23; c) M. W. P. L. Baars, E. W. Meijer, Top. Curr. Chem. 2000, 210, 131-182; d) G. R. Newkome, E. He, C. N. Moorefield, Chem. Rev. 1999, 99, 1689-1746; e) F. Zeng, S. C. Zimmerman, Chem. Rev. 1997, 97, 1681-1712.

[2] Dendrimers can be made using supramolecular interactions: a) S. C. Zimmerman, F. Zeng, D. E. C. Reichert, S. V. Kolotuchin, Science 1996, 271, 1095-1098; b) K. C.-F. Leung, F. Aricó, S. J. Cantrill, J. F. Stoddart, J. Am. Chem. Soc. 2005, 127, 5808-5810; c) W. T. S. Huck, L. J. Prins, R. H. Fokkens, N. M. M. Nibbering, F. C. J. M. van Veggel, D. N. Reinhoudt, J. Am. Chem. Soc. 1998, 120, 6240-6246; d) H. W. Gibson, N. Yamaguchi, L. Hamilton, J. W. Jones, J. Am. Chem. Soc. 2002, 124, 4653-4665.

[3] a) S. C. Zimmerman, Y. Wang, P. Bharathi, J. S. Moore, J. Am. Chem. Soc. 1998, 120, 2172-2173; b) A. M. Naylor, W. A. I. Goddard, G. E. Kiefer, D. A. Tomalia, J. Am. Chem. Soc. 1989, 111, 2339-2341; c) P. Walliman, P. Seiler, F. Diedrich, Helv. Chim. Acta 1996, 79, 779-788.

[4] a) N. Tomioka, D. Takasu, T. Takahashi, T. Aida, Angew. Chem. 1998, 110, 1611-1614; Angew. Chem. Int. Ed. 1998, 37, 1531-1534; b) S. Jockusch, N. J. Turro, D. A. Tomalia, Macromolecules 1995, 28, 7416-7418; c) B. Alonso, I. Cuadrado, M. Moran, J. Losada, Chem. Mater. 1995, 7, 1440-1442; d) Y. B. Lim, T. Kim, J. W. Lee, S. M. Kim, H. J. Kim, K. Kim, J. S. Park, Bioconjugate Chem. 2002, 13, $1181-1185$

[5] D. Banejee, M. A. C. Broeren, M. H. P. van Genderen, E. W. Meijer, P. L. Rinaldi, Macromolecules 2004, 37, 8313-8318.
[6] a) G. Magro, P. Marchand, R.-M. Sebastián, C. Guyard-Duhayon, A.-M. Caminade, J.-P. Majoral, Eur. J. Org. Chem. 2005, 1340-1347; b) J. W. Kriesel, S. Konig, M. A. Freitas, A. G. Marshall, J. A. Leary, T. D. Tilley, J. Am. Chem. Soc. 1998, 120, 12207-12215; c) A. W. Bosman, M. J. Bruining, H. Kooijman, A. L. Spek, R. A. J. Janssen, E. W. Meijer, J. Am. Chem. Soc. 1998, 120, 8547-8548.

[7] a) P. Brocorens, R. Lazzaroni, J.-L. Brédas, J. Phys. Chem. B 2005, 109, 19897-19907; b) T. Çağin, G. Wang, R. Martin, N. Breen, W. A. Goddard III, Nanotechnology 2000, 11, 77-84; c) L. Cavallo, F. Fraternali, Chem. Eur. J. 1998, 4, 927-934; d) C. B. Gorman, J. C. Smith, Polymer 2000, 41, 675-683; e) M. Han, P. Chen, X. Yang, Polymer 2005, 46, 3481-3488; f) K. J. Haxton, D. J. Cole-Hamilton, R. E. Morris, Dalton Trans. 2004, 1665-1669; g) C. Jana, G. Jayamurugan, R. Ganapathy, P. K. Maiti, N. Jayaraman, A. K. Sood, J. Chem. Phys. 2006, 124, 204719; h) H. Lee, J. R. Baker Jr. , R. G. Larson, J. Phys. Chem. B 2006, 110, 4014-4019; i) P. K. Maiti, T. Cağin, G. Wang, W. A. Goddard III, Macromolecules 2004, 37, 6236-6254; j) P. K. Maiti, T. Çağin, S.-T. Lin, W. A. Goddard III, Macromolecules 2005, 38, 979-991; k) W. Ortiz, A. E. Roitberg, J. L. Krause, J. Phys. Chem. B 2004, 108, 8218-8225; 1) S. Pricl, M. Fermeglia, M. Ferrone, A. Asquini, Carbon 2003, 41, 2269-2283; m) R. Scherrenberg, B. Coussens, P. van Vliet, G. Edouard, J. Brackman, E. de Brabander, K. Mortensen, Macromolecules 1998, 31, 456-461; n) N. Zacharopoulos, I. G. Economou, Macromolecules 2002, 35, 1814-1821.

[8] a) P. Miklis, T. Çăin, W. A. Goddard III, J. Am. Chem. Soc. 1997, 119, 7458-7462; b) G. Teobaldi, F. Zerbetto, J. Am. Chem. Soc. 2003, 125, 7388-7393.

[9] CCDC-648377-648382 contain the supplementary crystallographic data for this paper. These data can be obtained free of charge from The Cambridge Crystallographic Data Centre via www.ccdc.cam. ac.uk/data_request/cif.

[10] Crystals of $\mathbf{1}$ were grown by vapor diffusion of isopropyl ether into a solution of the acetate salt of the pincer in methanol and acetone and $\mathbf{2}$ by slow evaporation/cooling of a heated methanolic solution (wet) containing the pincer.

[11] X-ray quality single crystals were grown by vapor diffusion of isopropyl ether into a solution of $\mathbf{3}$ in methanol. The triethyl ammonium salt 3.TEA crystals were obtained by the vapor diffusion of isopropyl ether into an ethanol/acetone solution of the salt. The adamantyl guest $\mathbf{4}$ was recrystallized by vapor diffusion of isopropyl ether into a solution of $\mathbf{4}$ in a mixture of ethanol and chloroform.

[12] Crystals of $\mathbf{1}$ were obtained in our attempt to grow co-crystals of the pincer with acetic acid.

[13] a) A. Ballabh, D. R. Trivedi, P. Dastidar, E. Suresh, CrystEngComm 2002, 4, 135-142; b) B. L. Chen, S.-C. Ng, Y.-L. Feng, S.-X. Liu, Polyhedron 1998, 17, 4237-4247.

[14] The strength of dipole-dipole interactions is proportional to the inverse cube of the distance whereas that of ion-dipole is proportional to the inverse square of the distance.

[15] L. Kalé, R. Steel, M. Bhandarkar, R. Brunner, A. Gursoy, N. Krawetz, J. Phillips, A. Shinozaki, K. Varadarajan, K. Schulten, J. Comp. Physiol. 1999, 151, 283-312.

[16] S. Alavi, D. L. Thompson, J. Phys. Chem. A 2004, 108, 8801-8809.

[17] H.-Y. Liao, S.-Y. Chu, New J. Chem. 2003, 27, 421-424.

Received: April 13, 2007 Published online: July 5, 2007 